\title{
Using Action Plans to Help Primary Care Patients Adopt Healthy Behaviors: A Descriptive Study
}

\author{
Margaret Handley, PhD, MPH, Kate MacGregor, MPH, Dean Schillinger, MD, \\ Claire Sharifi, Sharon Wong, MPH, and Thomas Bodenheimer, MD
}

Purpose: An action plan is an agreement between clinician and patient that the patient will make a specific behavior change. The goals of this study are to: determine whether it is feasible for patients to make action plans in the primary care visit; determine whether patients report carrying out their action plans; and describe the action plans patients choose.

Methods: Forty-three clinicians in 8 primary care sites were recruited to hold action-plan discussions with patients. Research assistants contacted patients by telephone 3 weeks later to assess whether patients had conducted their action plans.

Results: Eighty-three percent of enrolled patients (228) made an action plan during a primary care visit. of the $79 \%$ who recalled making the action plan when interviewed by telephone 3 weeks later, $56 \%$ recalled the details of their action plan, and an additional $33 \%$ recalled the general nature of the action plan. At least 53\% of patients making an action plan reported making a behavior change consistent with that action plan.

Conclusions: Most patients reported making a behavior change based on an action plan, suggesting that action plans may be a useful strategy to encourage behavior change for patients seen in primary care. (J Am Board Fam Med 2006;19:224-31.)

Assisting patients to improve health-related behaviors is an important responsibility of primary care clinicians. ${ }^{1}$ However, physicians in primary care seldom have time to engage in behavior-change counseling and may be unsure how to discuss behavior change with their patients. ${ }^{2-4}$

One strategy to encourage behavior change involves a collaborative process in which patients choose a goal and clinicians and patients negotiate a specific action plan to assist in the goal's attainment. For example, the goal may be to lose 10 pounds; an initial action plan may be to substitute water in place of sugar-containing sodas. Patients

Submitted 7 September 2005; revised 7 November 2005; accepted 14 November 2005.

From the Department of Family and Community Medicine (MH, KM, CS, SW, TB), Division of General Internal Medicine (DS), University of California at San Francisco, San Francisco, CA.

Funding: This research was funded by the Robert Wood Johnson Foundation. DS was supported by a National Institutes of Health Mentored Clinical Scientist Award K-23 RR16539-03.

In this issue, see related article by MacGregor et al on page 215 and Commentary on page 324.

Conflict of interest: none declared.

Corresponding author: Thomas Bodenheimer, MD, Building 80-83, San Francisco General Hospital, 1001 Potrero Avenue, San Francisco, CA 94110 (E-mail: tbodenheimer@fcm.ucsf.edu). should have a high level of confidence that they can accomplish the action plan; success increases selfefficacy (a person's confidence that he/she can make positive life changes), and self-efficacy has been associated with healthier behaviors and improved clinical outcomes. ${ }^{5}$

Several studies suggest that collaborative goal setting and action planning may be more effective in promoting behavior change than traditional clinician-directed advice. ${ }^{6-8}$ However, no study offers details on action plans as a specific behavior-change intervention in primary care.

We performed a descriptive study of action plans in a diverse sample of primary care patients with coronary heart disease (CHD) risk factors. A companion article ${ }^{9}$ discusses the acceptability and feasibility of primary care clinicians engaging their patients in action-plan discussions. In this article, we explore the following questions. 1) To what extent are patients able to make an action plan with their clinician during a routine primary care visit? 2) What types of action plans do patients and clinicians make? 3) What proportion of patients are able to describe, and report carrying out, their action plans after 3 weeks? 4) Are certain patient characteristics associated with carrying out action plans? 


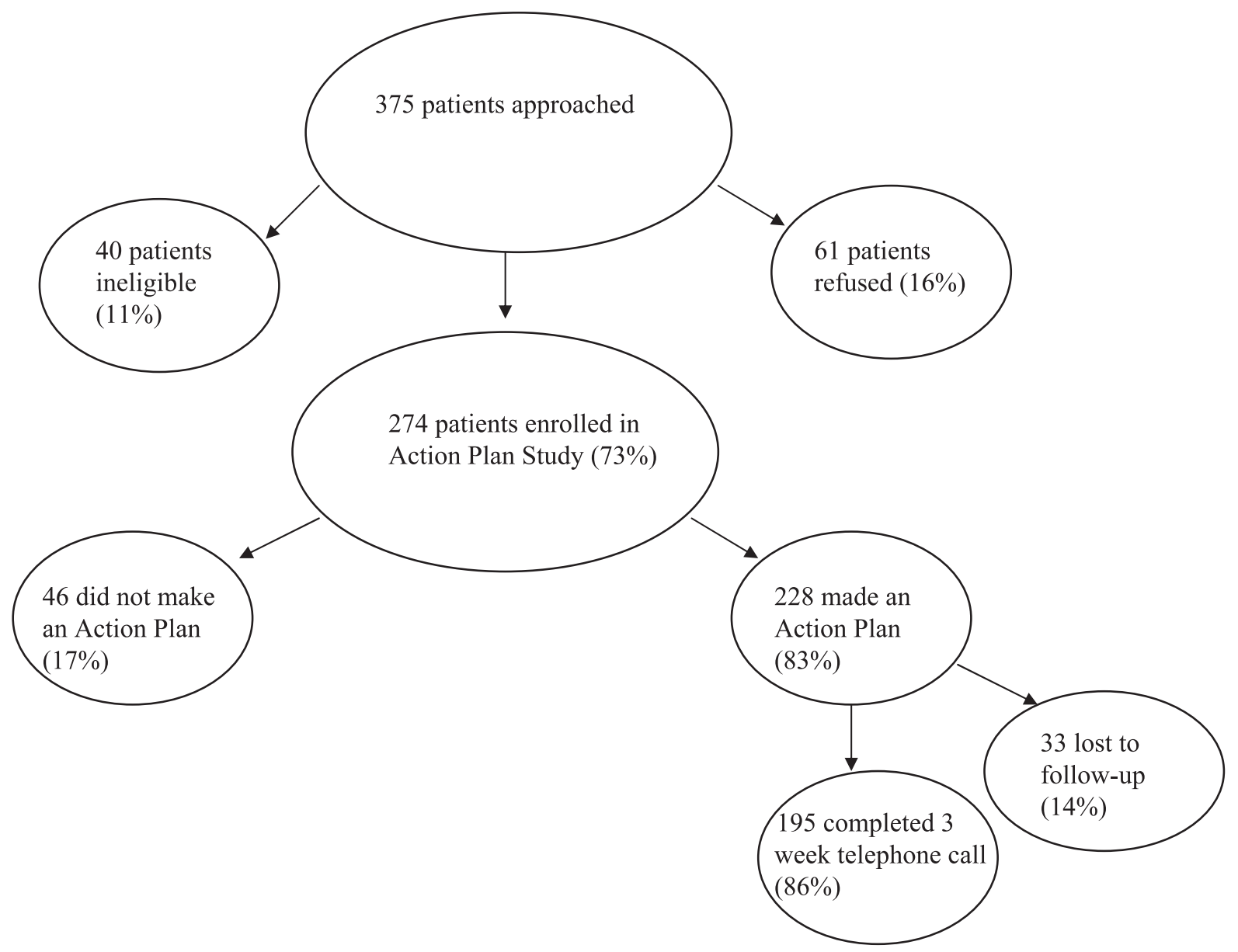

Figure 1. Participant Flow. Eligible patients with CHD risk factors defined as having a diagnosis of diabetes, CHD, hyperlipidemia, or hypertension; a clinical note indicating the patient was obese or overweight or a smoker; elevated blood pressure (diastolic $\geq 90 \mathrm{~mm} \mathrm{Hg}$ or systolic $\geq 140 \mathrm{~mm} \mathrm{Hg}$ ), or laboratory evidence of elevated lowdensity lipoprotein cholesterol $(>100)$ or diabetes (elevated hemoglobin A1C $>7.0)$ in the previous 3 months. Ineligible patients were those planning to leave the area or to change clinicians during the next 6 months, or who lacked access to a telephone. Patients were excluded following chart review and consultation with study clinicians if they had evidence of drug or alcohol addiction, serious mental illness, end-stage renal disease or cancer.

We had the following hypotheses regarding patient engagement in making action plans. Patients in a "precontemplative" stage of change, patients with low self-efficacy, and those receiving care at safety-net clinics would be less likely to make action plans than patients at a higher stage of readiness to make behavior changes, with greater self-efficacy, and receiving care in private practices.

\section{Methods}

\section{Recruitment}

In November 2003, we recruited 43 clinicians in 4 safety-net health centers and 4 private practices to engage in collaborative goal-setting discussions with at least 6 of their patients with CHD risk factors. Details of the practice and clinician recruitment, and of clinician training in action planning, are provided in the companion article. ${ }^{9}$

\section{Patient Recruitment}

Patients were enrolled between January and April of 2004. Trained research assistants reviewed patient charts to determine eligibility for patients with upcoming appointments. English-speaking adult patients were eligible based on having at least one ongoing CHD risk factor (see Figure 1 for details). Eligible patients were approached by a research assistant right before their clinic visit, 
asked to participate, and given informed consent. Enrolled patients were then interviewed by the research assistant before their clinician visit and asked questions about their demographic background, health status, recent health behaviors, stage of change/motivation to change behaviors, and self-efficacy regarding health-related behaviors. Demographic, health status, and recent health behavior measures were taken from the $2002 \mathrm{Be}-$ havioral Risk Factor Surveillance System survey; ${ }^{10}$ the motivation-to-change measure was based on the stages-of-change model ${ }^{11}$ and self-efficacy was measured using a 0 to 10 scale (with 10 being high self-efficacy) for health, diet, exercise, worry, stress, help, and overall confidence. ${ }^{12}$ If an action plan was made during the clinician visit, copies went to the patient, the patient's chart, and the research assistant.

Patients who made an action plan were told that the research assistant would follow-up with them in 3 weeks by phone. The telephone survey assessed whether patients remembered discussing an action plan with their clinician; patients were not prompted if they did not remember. Those who did remember making an action plan were asked to describe it, and asked whether they were carrying it out. The study was approved by the University of California at San Francisco (UCSF) Institutional Review Board.

\section{Action Plan Coding}

Two researchers (CS and $\mathrm{MH}$ ) independently coded the action plans; disagreements were discussed with other research team members to achieve consensus. Coding was done within action plans domains (subcategories for diet, physical activity, medication, smoking, stress, and "other") and for level of specificity (using a scale of 1 to 3 , with 1 being not specific beyond the domain; 2 being somewhat specific regarding the description of the action to be undertaken or about the time frame/ frequency of the action, but not both; and 3 being bighly specific to both the action to be undertaken and the time frame/frequency of completing it). Additionally, action plans were coded to distinguish between those describing a one-time event and an on-going activity.

Open-ended responses from the telephone interviews were also coded. Action plan descriptions were coded as matching on domain and details, matching on domain alone, or not matching on either. Patient responses to the question, "How are you doing with your action plan?" were coded into two categories, "completed" or "not completed" to characterize whether patients were carrying out the behavior change described in the action plan.

\section{Data Entry and Analysis}

Data were entered into ACCESS and analyzed with SAS (SAS Institute, Cary, NC). Characteristics of patients who did and did not make action plans were compared using $\chi^{2}$, Fisher's exact, and $t$ tests. To examine the association between baseline patient characteristics and action plan completion at a 3-week follow-up (completed versus did not complete action plan), multivariate regression analyses were done, including analyses using generalized estimating equations to account for the potential patient clustering of responses within clinician.

\section{Results}

Three hundred seventy-five patients were approached for the study of whom 40 (11\%) were ineligible, 61 declined (16\%), and 274 were enrolled (73\%) (Figure 1).

\section{Patient Characteristics}

Patients were racially and ethnically diverse. Fortyseven percent of enrolled patients attended safetynet clinics and $53 \%$ private practice settings (Table 1). Thirty-six percent reported being in fair or poor health. Most had multiple CHD risk factors (86\%). The majority of patients (69\%) were in the action/ maintenance stage of change at the initial visit (reporting they had made changes to improve their health in the last 6 months), with fewer patients (7\%) determined to be precontemplative (reporting they had made no health-related changes in the past 6 months and were not intending to in the next 6 months).

\section{Action Plans during Primary Care Visits}

Eighty-three percent of patients made an action plan with their clinician the day they enrolled in the study. Comparisons between patients who did and did not make action plans indicated few differences. Eighty-two percent of patients in safety-net clinics made action plans compared with $84 \%$ in private practices, with no statistical difference. Of the precontemplative patients, $70 \%$ made an action plan at the initial visit, with no statistical difference in the 


\begin{tabular}{|c|c|c|c|}
\hline \multirow[b]{2}{*}{ Variable/Definition } & \multicolumn{3}{|c|}{ Mean or \% (SD) } \\
\hline & $\begin{array}{l}\text { All enrolled } \\
\text { patients }(\%) \\
(\mathrm{n}=274)\end{array}$ & $\begin{array}{l}\text { Patients making } \\
\text { action plans }(\%) \\
\quad(\mathrm{n}=228)\end{array}$ & $\begin{array}{l}\text { Patients not making } \\
\text { action plans }(\%) \\
(\mathrm{n}=46)\end{array}$ \\
\hline \multicolumn{4}{|l|}{ CHD risk factors* } \\
\hline Diabetes & 31 & 32 & 22 \\
\hline Hyperlipidemia & 48 & 49 & 44 \\
\hline Hypertension & 63 & 61 & 70 \\
\hline Overweight & 62 & 64 & $48 \dagger$ \\
\hline Coronary heart disease & 7 & 7 & 9 \\
\hline Smoking & 27 & 28 & 20 \\
\hline Multiple CHD risk factorsł & 86 & 86 & 87 \\
\hline Age (mean years, SD) & $52(12.7)$ & $52(13.1)$ & $54(10.8)$ \\
\hline \multicolumn{4}{|l|}{ Race/ethnicity } \\
\hline African American & 33 & 35 & 24 \\
\hline White & 30 & 29 & 35 \\
\hline Latino/a & 10 & 10 & 7 \\
\hline Asian American & 16 & 14 & $28 \dagger$ \\
\hline Other & 11 & 12 & 6 \\
\hline \multicolumn{4}{|l|}{ Education } \\
\hline High School or less & 42 & 43 & 39 \\
\hline \multicolumn{4}{|l|}{ Clinic setting } \\
\hline Public & 47 & 46 & 50 \\
\hline Private & 53 & 54 & 50 \\
\hline \multicolumn{4}{|l|}{ Health status $\$$} \\
\hline Fair or poor & 36 & 36 & 36 \\
\hline Good & 39 & 38 & 43 \\
\hline Very good or excellent & 25 & 26 & 21 \\
\hline \multicolumn{4}{|l|}{ Health risk factors $\|$} \\
\hline Physical inactivity (none) & 15 & 15 & 17 \\
\hline Skipped medication $(1+$ times/week $)$ & 19 & 20 & 13 \\
\hline Few fruits/vegetables ( $\leq 2$ per week) & 31 & 33 & 22 \\
\hline \multicolumn{4}{|l|}{ Stage of changeף } \\
\hline Precontemplative & 7 & 6 & 13 \\
\hline Contemplative & 2 & 2 & 2 \\
\hline Preparation & 22 & 23 & 17 \\
\hline Action/maintenance & 69 & 69 & 68 \\
\hline \multicolumn{4}{|l|}{ Self-efficacy ${ }^{* *}$ (mean score, SD) } \\
\hline Health confidence & $8.5(2.2)$ & $8.6(2.1)$ & $8.2(2.5)$ \\
\hline Diet confidence & $7.6(2.6)$ & $7.5(2.7)$ & $7.8(2.6)$ \\
\hline Exercise confidence & $7.0(2.7)$ & $6.9(2.8)$ & $7.5(2.5)$ \\
\hline Worry confidence & $5.7(3.1)$ & $5.6(3.1)$ & $6.1(3.1)$ \\
\hline Stress confidence & $6.6(3.1)$ & $6.5(3.1)$ & $6.8(3.2)$ \\
\hline Help confidence & $8.0(2.4)$ & $8.0(2.3)$ & $7.8(3.1)$ \\
\hline Overall confidence & $8.1(2.6)$ & $8.1(2.6)$ & $8.1(2.6)$ \\
\hline
\end{tabular}

* Based on chart review prior to study enrollment using the following criteria: (1) diagnosis of diabetes, hypertension, coronary heart disease, hyperlipidemia; (2) clinical note indicating patient is overweight or a smoker; (3) recent laboratory report indicating elevated blood pressure (dystolic $\geq 90$ or systolic $\geq 140$ ), elevated low-density lipid $(>100)$ or hemoglobin Alc value $>7.0$.

$\dagger P<.05$ for comparisons between patients making action plans $(\mathrm{n}=228)$ and patients not making action plans $(\mathrm{n}=46)$.

$\neq$ Presence of 2 or more CHD risk factors in chart review.

$\S$ Based on the Risk Factor Surveillance System survey. ${ }^{10}$

$\|$ In the previous month.

II Based on the transtheoretical model. ${ }^{11}$ Precontemplative: have not made a behavior change in the past 6 months to improve health and are not planning any such changes in the next 6 months. Contemplative: have not made any changes in the past 6 months but are planning to in the next 6 months. Preparation: have made no changes in the past 6 months but plan to in the next 30 days. Action/maintenance: have made changes in the past 6 months to change health-related behaviors.

** Scale 0 to 10 . Higher score is better. Responses to: How sure are you that you can (behavior)? Health confidence: do the different tasks and activities needed to manage your illness? Diet confidence: make changes in your diet to improve your health? Exercise confidence: make and stay with a regular exercise program. Worry confidence: keep tiredness, pain, or worry from interfering with things you want to do? Stress confidence: remain calm when faced with difficulties related to your health? Help confidence: find the help you need when having problems with your health or your life? Overall confidence: solve most of your problems if you try hard enough? 
Table 2. Description of Baseline Action Plans $(\mathrm{N}=228)$

\begin{tabular}{|c|c|c|}
\hline Action Plan Domains and Subtopics & $\mathrm{N}$ & $\%$ \\
\hline Exercise $(38 \%)$ & 86 & \\
\hline Walk a specific distance or specific amount of time & 46 & 53 \\
\hline Use gym more, more of any exercise & 37 & 43 \\
\hline Buy a pedometer & 3 & 4 \\
\hline Diet $(30 \%)$ & 68 & \\
\hline Eat more good food, substitute good food for bad & 24 & 35 \\
\hline Portion control of bad foods & 27 & 40 \\
\hline Lose weight/go on a diet & 6 & 9 \\
\hline Other (eg, see nutritionist, track eating, change others' behaviors) & 11 & 16 \\
\hline Combined diet and exercise* $(4 \%)$ & 9 & 4 \\
\hline Medication (7\%) & 16 & \\
\hline Take specific medication & 5 & 31 \\
\hline Take all meds & 6 & 38 \\
\hline Take meds at specific time & 3 & 19 \\
\hline Take vitamins, etc & 2 & 12 \\
\hline Other† $(9 \%)$ & 21 & \\
\hline Make/keep appointments & 5 & 25 \\
\hline Socialize or join group & 3 & 15 \\
\hline Initiate positive action & 9 & 45 \\
\hline Increase self-monitoring & 3 & 15 \\
\hline Smoking (8\%) & 19 & \\
\hline Reduce smoking frequency (episodes or no. of cigarettes) & 11 & 58 \\
\hline Quit smoking/patch to quit & 7 & 37 \\
\hline Track smoking & 1 & 5 \\
\hline Stress $(4 \%)$ & 9 & \\
\hline Time with others & 2 & 22 \\
\hline Structured relaxation exercises & 4 & 45 \\
\hline Reading & 2 & 22 \\
\hline Time by self for self & 1 & 11 \\
\hline
\end{tabular}

* A combined diet/exercise domain was added during the coding process. Most patients making action plans reported a high level of confidence that they could complete their plan-79\% had a confidence score $>7$.

† One Action Plan is illegible in this category and not coded.

proportion of patients who were precontemplative compared with patients in other stages of change $(84 \%)$, regarding having made an action plan during the primary care visit $(P>.10)$. There were no differences in mean self-efficacy measures for patients who did and did not make action plans, or for the proportion who had higher versus lower selfefficacy scores (eg, scoring $\geq 7$ out of a 0 to 10 confidence scale versus 6 or lower) (84\% versus $83 \%)$.

\section{Action Plan Descriptions}

Table 2 summarizes patients' action plans across the 6 domains listed on the action plan form. Over two thirds of the action plans were in the domains of diet or physical activity.
Some examples of exercise action plans include: "walk around apartment complex everyday," "call YMCA for water aerobics class," and "dance for 15 minutes every day." Examples from diet-related action plans include: "reduce bread from 2 slices to 1 slice 3 times a day," "put leftover food away in the fridge before eating meal," and "no chocolate on Tuesday and Thursday." Examples of smokingrelated action plans include: "not lighting up after going to and from work," "make a list of pros and cons for smoking," and "quit buying cigarettestoday!" One medication action plan was: "take a container with evening meds in my back pack." "Doing something I need to work on" examples include: "ride bus with friend 3 times a week," "join diabetes group," and "talk to my sister and brother 
about telling my nieces and nephews not to buy me candy anymore."

Sixty-six percent of action plans were highly specific, indicating both the action and time period ("don't eat fried chicken more than once a week"). Twenty-one percent were coded as less specific (without details on the frequency or time period of the action, for example, "walk around the park" or "get the nicotine patch"), and $12 \%$ were nonspecific ("take medications" or "start exercising again"). The majority of action plans (78\%) were ongoing, for example, "walk around my apartment complex everyday" compared with one-time ("call a therapist"). Smoking and other action plans were less frequently ongoing ("call 1-800-no-butts" or "join a neighborhood group") compared with other domains $(51 \%$ versus $84 \%, P<.001)$.

\section{Recall of Action Plans at 3-Week Follow-up}

Of the 228 study participants who made an action plan with their clinician, 195 (86\%) were interviewed by telephone 3 weeks after the visit (Figure 1). Of these, 180 (92\%) reported they recalled making an action plan with their clinician. Only patients who recalled making an action plan were asked to describe it. Of these 180 patients, 101 (56\%) described their action plan with both the domain and details matching the original action plan. An additional 60 patients (33\%) reported the same domain as the original action plan but not the details, and 19 patients (11\%) reported different domains and details from that recorded from their study visit.

Among patients who reported recalling their action plans at follow-up and who provided matching action plan domain and/or details $(n=161)$, the majority (75\%) also reported that they had been carrying out their action plan in the preceding 2 to 3 weeks. The domains with the highest action plan completion were: diet/exercise (88\%) and diet (85\%), followed by other (88\%), medication (78\%), exercise (74\%), smoking (67\%), and stress (67\%). Based on these reports and the assumption that patients we were not able to contact for follow-up or who did not remember the details of their action plan did not make the proposed behavior change, we conservatively estimate that at least $53 \%$ of all patients who made an action plan at their initial visit $(\mathrm{n}=228)$ had completed/conducted the behavior change by 3 weeks.
The following exploratory analyses were adjusted for potential clustering of patient responses within clinicians. Since there were no statistical differences between the adjusted and unadjusted results, either for possible clinician clustering or for the addition of covariates to the models, only the unadjusted results are presented. None of the patient characteristics hypothesized to be associated with completing an action plan was significantly associated with action-plan completion at followup, although the sample size was small for these analyses $(n=155)$. Seventy-eight percent of patients in both the precontemplative stage and in other stages of change reported carrying out their action plan, suggesting that patients who were not planning to make a health-related change in the next 30 days were as likely to carry out action plans as those reporting a greater degree of motivation. Similarly, with regards to the proportion of patients who reported carrying out their action plans, there were no significant differences between patients who attended safety-net clinics (75\%) versus those seen in private practices $(80 \%)$, or for patients reporting poor or fair health $(80 \%)$ versus good, very good or excellent health (77\%). There were no differences in the baseline mean self-efficacy scores of patients who did and did not carry out their action plan at follow-up; similarly, $80 \%$ of patients with baseline self-rated self-efficacy of 7 or greater conducted an action plan compared with $70 \%$ of those with scores of 6 or below.

Characteristics of the action plans were generally not associated with carrying out an action plan at follow-up, although more patients with ongoing action plans reported carrying them out compared with patients with one-time action plans (81\% versus $66 \%, P=.06$ ).

\section{Discussion}

In this study of diverse primary care patients with CHD risk factors and high levels of co-morbidity cared for in a number of clinical settings, we found that (1) the majority of patients and clinicians were able to make behavior-change action plans in the primary care visit, (2) most patients could accurately describe the action plan they made a few weeks later, and (3) over two-thirds of those who could describe their action plan reported making at least some of the proposed behavior changes. Assuming that all non-respondents to the follow-up 
interview did not do their action plan, over half the participants making an action plan at their initial visit reported that they were able to carry out their chosen health-behavior change over a short time period.

Our findings suggest that collaborative goal-setting with action plans may be a useful strategy to encourage behavior change for primary care patients. That study participants were selected through a chart review process rather than through clinician referral, that most patients with CHD risk factors were eligible, and because the refusal rate was relatively low, it is likely that patients enrolled in this study are similar to patients not enrolled in the study. However, we did not obtain comparative data on non-enrolled patients to determine to what extent these findings are applicable to the broader patient population.

An important clinical lesson suggested from this study concerns directive versus collaborative decision making. Traditionally, clinicians tell patients what to do, and direct patients in a general rather than a specific way: "Your cholesterol is too high;" "You need to lose weight." The collaborative approach allows the patient to choose the domain of behavior change, and the action plan creates a specific, rather than a vague and general, program of behavior change. ${ }^{13}$ While not designed to demonstrate whether collaborative goal-setting using action plans, compared with clinician-directed advice, is associated with greater behavior change, this study does suggest that patients from a variety of clinical settings are willing to engage in the collaborative process.

It is commonly believed that patients vary in their readiness to make behavior changes and that behavior-change strategies should be tailored to an assessment of "stage of change." 11 We hypothesized that patients assessed to be "precontemplative" would be less likely to carry out an action plan. Our study suggests that patients in the precontemplative stage can be engaged in action-plan discussions and that they report carrying out their action plans with similar frequency to patients expressing greater readiness to change. It has been reported that practitioners sometimes ignore unhealthy behaviors in precontemplative patients. ${ }^{14}$ This misinterpretation of the "stage-of-change" concept could lead clinicians to avoid action-plan discussions with patients who report little motivation to change because they believe the patients will fail. It is possible that patients lack motivation to make healthy changes because they fear failure. Our study suggests that the action plan processagreeing on a small change with a high probability of success-may convert some precontemplative patients to the action stage. Similarly, patients in our study who initially had a low level of confidence that they could adopt healthy behaviors (low selfefficacy) made and conducted action plans as often as those with high self-efficacy scores.

We also hypothesized that patients in safety-net clinics-because they frequently face many life challenges related to their socio-economic statuswould have a harder time changing behaviors than patients in private practices. Our study demonstrates, however, that safety-net patients made action plans and carried them out with the same frequency as private practice patients. Clinicians should not assume that lower income patients in a primary care setting are less able to engage in collaborative goal setting to promote behavior change.

\section{Limitations}

Our study has a number of limitations. It was not designed to answer the question: "Do patients engaging in action-plan discussions in primary care have improved behaviors and clinical outcomes compared with patients receiving usual care?" Neither does this study address whether patients who reported carrying out their action plans continue to do so over a longer time period. We were not able to determine the validity of patient reports on carrying out their action plans and it is possible that patients over-reported their behavior changes.

The sample size for the study was small and further research is needed to confirm the results of our exploratory analyses. Larger studies are needed to test the conclusions regarding our initial hypotheses, which is that patients assessed as "precontemplative," those with low self-efficacy before the action-plan discussion, and those receiving care at safety-net clinics were as likely to make and carry out action plans as patients at a higher stage of readiness, with greater self-efficacy, and receiving care in private practices.

Finally, the study design required that patients be enrolled by a research assistant before their visit and that clinicians try to engage in action-plan discussions with patients that same day. Neither condition reflects primary care reality; patients and 
clinicians may have been more likely to attempt action-plan discussions under study conditions than in real-world practice. Alternatively, patients and clinicians might be more likely to engage in actionplan discussions when artificial conditions are not present.

\section{Conclusion}

Among primary care patients with one or more coronary heart disease risk factors who are encouraged to generate a behavioral action plan, a majority make an action plan and report making a behavior change based on that action plan. These results suggest that incorporating collaborative goal setting with action plans into routine primary care practice may be a useful strategy to promote behavior change. We believe that the next step in generating new knowledge about collaborative goal-setting and action planning should be a randomized controlled trial of action planning versus usual care with a long follow-up period, measuring both self-reported behavior change and objective clinical outcomes.

\section{References}

1. Whitlock EP, Orleans CT, Pender N, Allan J. Evaluating primary care behavioral counseling interventions. Am J Prev Med 2002;22:267-84.

2. Ma J, Urizar GG Jr, Alehegn T, Stafford RS. Diet and physical activity counseling during ambulatory care visits in the United States. Prev Med 2004;39: 815-22.

3. Rosal MC, Ockene JK, Luckmann R, et al. Coronary heart disease multiple risk factor reduction. Providers' perspectives. Am J Prev Med 2004;27(2 Suppl): 54-60.

4. Yarnell KS, Pollak KI, Ostbye T, Krause KM,
Michener JL. Primary care: is there enough time for prevention? Am J Public Health 2003;93:635-41.

5. Marks R, Allegrante JP, Lorig K. A review and synthesis of research evidence for self-efficacy-enhancing interventions for reducing chronic disability (Parts 1 and 2). Health Promotion Practice 2005;6: 37-43, 148-56.

6. Cullen KW, Baranowski T, Smith SP. Using goal setting as a strategy for dietary behavior change. J Am Diet Assoc 2001;101:562-6.

7. Ammerman AS, Lindquist CH, Lohr KN, Hersey J. The efficacy of behavioral interventions to modify dietary fat and fruit and vegetable intake: a review of the evidence. Prev Med 2002;35:25-41.

8. Shilts MK, Horowitz M, Townsend MS. Goal setting as a strategy for dietary and physical activity behavior change: a review of the literature. Am J Health Promot 2004;19:81-93.

9. MacGregor K, Handley M, Wong S, et al. Behaviorchange action plans in primary care: a feasibility study of clinicians. J Am Board Fam Med 2006;19: 215-23.

10. Centers for Disease Control and Prevention (CDC). Behavioral Risk Factor Surveillance System Survey Questionnaire. Atlanta (GA): U.S. Department of Health and Human Services, Centers for Disease Control and Prevention; 2002.

11. Prochaska JO, Velicer WF. The transtheoretical model of health behavior change. Am J Health Promot 1997;12:38-48.

12. Lorig K, Stewart A, Ritter P, Gonzalez V, Laurent D, Lynch J. Outcome measures for health education and other health care interventions. Thousand Oaks (CA): Sage Publications; 1996.

13. Bodenheimer T, Lorig K, Holman H, Grumbach K. Patient self-management of chronic disease in primary care. JAMA 2002;288:2469-75.

14. Rollnick S, Mason P, Butler C. Health behavior change: a guide for practitioners. Edinburgh: Churchill Livingstone; 2000. p. 187-8. 